\title{
Demands on Shape Memory Alloys from the Application Designer's Point of View
}

\author{
P. Abrahamsson and E. Møster \\ Department of Machine Design, Lund Institute of Technology, Box 118, 22100 Lund, Sweden
}

\begin{abstract}
In order to improve the conditions for utilization of Shape Memory Alloys (SMAs) in engineering design, the engineering design process and the shape memory effect have been studied from the viewpoint of the engineering designer. Further, the maturity of the SMA technology has been evaluated according to a common evaluation technique denoted the technology readiness assessment. The results obtained in this paper confirm that SMAs introduce unacceptable uncertainties in the engineering design process. It is shown that the SMA technology is still not sufficiently mature to be utilized in engineering design. Based on the requirement that the SMA technology must be upgraded to a level satisfying the conditions set by the technology readiness assessment, this paper presents fundamental objectives for future research involving a closer collaboration between researchers from the fields of material technology and engineering design.
\end{abstract}

\section{INTRODUCTION}

Although it is 65 years since the young Swedish Rockefeller Scholarship holder Arne Ölander [1] first reported his observations of unusual "rubber-like" properties in a gold-cadmium alloy, it is first during the last decades that Shape Memory Alloys (SMAs) have been subjected to major research activities. So far, the research mainly has been focused on metallurgical problems concerning relations between stress, strain and temperature, as well as stability and materials processing. Despite the fact that major efforts have been made to utilize the shape memory effect in engineering design, remarkably few products are commercially available.

A few years ago, an information and knowledge inventory study on the utilization of SMAs in engineering design was started at the Department of Machine Design, Lund Institute of Technology. In a preliminary study, Abrahamsson [2] found that information and knowledge regarding SMAs are either lacking or not available in a form that fulfills the needs of the engineering designer. These findings were later confirmed by the results of an international survey by Abrahamsson and Bjärnemo [3]. The results verified the need for better information and knowledge of SMAs - as well as a need for complementary design tools. As a result of these findings, Bjärnemo and Burman [4] demonstrated an implementation procedure for modelling the shape memory effect in a general finite element code.

In an application study, Møster [5] showed that the selection of SMA must be performed at an early stage of the design process, to fully establish the working principle of the product to be. In a study by Burman et al. [6], it is shown that in order to facilitate a clear understanding and modelling of the working principle, complementary design tools, supporting the non-linear and time dependent properties of functional (intelligent) materials like SMAs, should be implemented at an early stage of the engineering design process.

Motivated by the findings from these studies, the main objective of this paper is to contribute to an increased use of SMAs by focusing on the needs of the engineering designer. The secondary objective is to investigate in which direction the development of the SMA technology ought to be directed in order to facilitate the needs imposed by the engineering design process.

The paper is structured into five sections. In order to get a basic understanding of the design related behavior of SMAs, the engineering design process is described in the light of common design procedure models in section 2. Based on the survey by Abrahamsson and Bjärnemo [3], the requirements for increasing the use of SMAs as identified in industry and by researchers in the field, are outlined in section 3 . In section 4 , a discussion is given on what actions should be taken in order to contribute to the maturity of the SMA technology. The final section summarizes the findings of the paper. 


\section{THE ENGINEERING DESIGN PROCESS}

Accelerating demands on companies to launch new and better products faster, with higher quality and at lower cost, are forcing most companies to use efficient approaches to the engineering design process (design process for short). This implies that the design of a mechanism based on the shape memory effect cannot be separated from the design of the product in which the mechanism is to be utilized.

In the study by Burman et al. [6] it is argued that, unlike conventional engineering materials, functional materials like SMAs do not only possess the ability to perform a passive function (i.e. supporting, stiffening or connecting) but also an active function (i.e. changing shape or material properties). This indicates the possibility that one single shape memory element could fulfil the same task as an assembly of several mechanical components. In the given context, the active function is in focus. To facilitate the understanding and modelling of the shape memory behavior, it should be noted that the close links between shape, dimension and material properties must be fully determined in order to define the active function of interest.

\subsection{General aspects of the engineering design process}

During the last decades, major efforts have been made to provide general systematic approaches to the design process by means of design procedure models, such as the ones by Asimov [7], Hubka and Eder [8], Olsson [9], Pugh [10], Pahl and Beitz [11], and Ullman [12]. A common denominator in these models is the structuring of the design process by means of phases, which in turn are decomposed into basic sub-activities. The models give the designer powerful tools to plan what to do and when to do it. Although the sequences of subactivities in the phases might differ significantly between different models, the majority of these are based on problem solving concepts involving the sub-activities of analysis, synthesis, evaluation, decision making and implementation.

The design process is a learning process, ranging from the abstract formulation of the design task to a concise description of the product to be. Starting at a low level of knowledge, the designer systematically gains increased knowledge of the product when performing each phase. Throughout the process, the conception of the product transforms from an idea to a physical product by way of mental, verbal, analytical and graphical models. Similarly, the design techniques and design methods utilized change from qualitative to quantitative.

During the design process, the design freedom is gradually decreased by decisions made by the designer. Simultaneous with the evolution of increased insight and understanding of the design task and its potential solutions, economical resources are consumed. As the understanding and knowledge of the design problem is low in the early phases, there always is a risk that wrong decisions might occur. A wrong decision in the early phases might be fatal to the outcome of the process if not discovered and changed through an iteration back to the phase in which the wrong decision was made. Obviously, such decisions should be avoided. Therefore, decisions regarding fundamental issues, such as the working principle of the product, must be made early in the process, whereas decisions regarding the selection of materials, form and dimensions are made at later stages.

\subsection{Utilization of SMAs in engineering design}

Early in the design process the designer creates a number of working principles based on different technical solutions (i.e. solutions based on hydraulics, pneumatics, and the shape memory effect), from which the best concept is to be selected through evaluation against the criteria established at the beginning of the design process. Hereby, the designer needs to perform some preliminary analysis. However, the worthiness of design concepts utilizing SMAs cannot be assessed without first determining the material properties, the shape, and the dimensions of the selected alloy. Consequently, to be able to analyze a working principle in which the shape memory effect is utilized, the designer needs to significantly increase his or her understanding of those factors which control the behavior of the SMA.

Obviously, this does not favour the utilization of SMAs in engineering design. To obtain these insights, the designer presently has to rely on experimental investigations which are both time and cost consuming. The obvious risk facing the designer is that the desired function cannot be accomplished by utilizing a working principle based on the SMA technology. In order to avoid this risk, a natural decision by the designer is to reject the SMA concept without even investigating it, for the benefit of a concept based on a more conventional technology. 


\section{REQUIREMENTS FOR FACILITATING THE USE OF SMAS}

The international survey previously mentioned was performed in two steps. In the first step a questionnaire, focusing on SMAs and SMA applications, was distributed to 135 addresses (researchers, engineers, companies and institutes with interest in SMAs) in 16 countries. Important questions were in what kinds of activities and applications SMAs were used and how the products were developed.

Replies were received from 53 addresses (39\%). To those who answered the first questionnaire, a second questionnaire was sent in order to establish what knowledge and information was available when using SMAs in engineering design. Especially, what problems did the choice of SMA technology introduce in the design process as well as in the subsequent life phases of the product?

The results of the survey indicate that a large series of requirements must be fulfilled to benefit an increased use of SMAs in engineering design. With respect to requirements concerning information, material, performance, design tools, operation and standardization, the findings are presented in table 1.

Table 1: Requirements for an increased use of SMAs (adapted from Abrahamsson and Bjärnemo [3])

\begin{tabular}{|c|c|c|}
\hline \multirow[t]{3}{*}{ I - Information: } & $\left(I_{1}\right)$ & $\begin{array}{l}\text { Extensive and reliable material property data provided by the manufacturer - especially on } \\
\text { transformation temperatures, applicable force, hysteresis, and fatigue life. }\end{array}$ \\
\hline & $\left(\mathrm{I}_{2}\right)$ & $\begin{array}{l}\text { Literature offering more detailed descriptions of product development projects utilizing } \\
\text { SMAs. }\end{array}$ \\
\hline & $\left(\mathrm{I}_{3}\right)$ & Networks involving designers and metallurgists for discussions and advice. \\
\hline \multirow[t]{3}{*}{ M-Material: } & $\left(\mathrm{M}_{1}\right)$ & $\begin{array}{l}\text { A wide range of commonly available materials providing different ranges of transformation } \\
\text { temperatures. }\end{array}$ \\
\hline & $\left(\mathrm{M}_{2}\right)$ & $\begin{array}{l}\text { More stable material properties - especially those concerning the functional behavior, such as } \\
\text { fatigue, hysteresis, transformation, ageing and deterioration temperatures. }\end{array}$ \\
\hline & $\left(\mathrm{M}_{3}\right)$ & Materials offering low hysteresis, high working temperatures and high energy efficiency. \\
\hline \multirow[t]{2}{*}{ P-Performance: } & $\left(P_{1}\right)$ & $\begin{array}{l}\text { Better understanding of the phenomenological (macroscopic) behavior and properties - struc- } \\
\text { tural as well as functional. }\end{array}$ \\
\hline & $\left(P_{2}\right)$ & $\begin{array}{l}\text { Constitutive equations describing the material behavior, such as relations between stress, } \\
\text { strength, temperature, hysteresis, fatigue etc. }\end{array}$ \\
\hline
\end{tabular}

D-Design tools:

$\left(D_{1}\right)$ Design tools describing the functional behavior, such as computerized constitutive material models, product geometry models and material property databases.

$\left(D_{2}\right)$ Design guidelines, such as guidelines regarding overheating protection, fatigue protection, control of operating temperature, joule heating and cooling.

$\left(\mathrm{D}_{3}\right)$ Design procedure models more properly benefiting utilization of SMAs.

O-Operation: $\quad\left(\mathrm{O}_{1}\right)$ Adaptive systems measuring the changes of material properties or product behavior and adapt-
ing the operational conditions to the properties and / or behavior changed.

S-Standardization: $\quad\left(S_{1}\right)$ Standardized materials.

$\left(S_{2}\right)$ Standardized methods for testing phenomenological properties.

$\left(S_{3}\right)$ Standardized methods for joining, soldering and welding.

An interesting result of the study was that only a few of the participating companies had made any attempt at formalizing design methods or techniques for SMAs. The documentation available on product development projects involving SMA-based products was rather poor. A common problem was that the material seldom behaved as specified by the manufacturer - especially in those cases where the SMA was manufactured simultaneous with the development of the product in which the SMA was to be utilized. The latter seemed to happen rather often.

In general, the information offered by the manufacturer proved to be insufficient and unreliable. Also, the study clearly indicated that the knowledge about how to make use of SMAs in engineering design was insufficient and that the availability of proper design tools was poor. Apart from the fraction of information available in literature, most information and knowledge of SMAs seems to come from own practice. Only three of the answers indicated that no additional knowledge was desired. 
Even though many participants in the survey were involved in product development projects, in which SMAs were utilized, the requirements identified in table 1 support the assumption that research on shape SMAs has been performed with focus on metallurgical aspects. Although communication is frequent between engineering designers and manufacturers of alloys, the results obtained indicate that cooperation between researchers from the fields of material technology and engineering design is very rare, if existing at all. If we aim to facilitate an increased use of SMAs in engineering design, the need for collaboration is urgent.

\section{DISCUSSION}

In order to investigate which actions should be taken in the development of a SMA technology more properly benefiting utilization in engineering design, the maturity of the SMA technology is discussed below according to an evaluation technique by Ullman [12] and the findings reported in the previous sections.

\subsection{Evaluation of the SMA technology}

In engineering design it is important that the technology to be utilized is sufficiently mature so that the fulfillment of the product task becomes a design issue and not a research topic. Utilization of immature technologies might result in unreliable products of low quality. According to Ullman's technology readiness assessment [12], a technology is mature and ready to be employed in a product when the following conditions are satisfied:

1. The product must be manufactured with known processes.

2. The critical parameters that control the function must be identified.

3. The safe operation latitude and sensitivity of the parameters must be known.

4. The failure modes have to be identified.

5. Hardware that verifies that the above four items are fulfilled must exist.

6. The technology must be controllable throughout the lifetime of the product.

None of the conditions outlined above are fully satisfied for the SMA technology. The first condition is poorly satisfied - SMAs can be manufactured with known processes, but the material properties are not sufficiently exact and reliable to be used in a design process without preceding, time-consuming and expensive, material tests. This seems to be due to sensitivity to the chemical composition, to the production process and to the subsequent processing.

The second condition says that parameters critical to a safe operation of the product must be known. To some extent this is satisfied - there do exist a general knowledge about the dependence between the most critical parameters (i.e. temperature, dimensions, and chemical composition) and functions depending on stress/strain relations, hysteresis and fatigue. However, this knowledge is mainly empirical and based on extensive tests. There are only a few theories available that can be used for analysis purposes in engineering design.

This statement is also valid for the third and fourth condition. In most cases, only qualitative information or very rough data for certain loading conditions are available on safe operating latitudes during the life of operation for SMAs. A Failure Mode and Effect Analysis (FMEA) is a technique for assessing the consequences of different failure modes. Failure modes are changes of operation conditions or errors made during design and manufacturing that can lead to failure of the product. To identify the failure modes and what actions to take, reliable relations between changes, errors and product operation must exist. As previously argued, the availability of such relations is poor within the SMA field.

Only a limited number of applications have proven successful on the market. In general, these are single cycle applications (i.e. pipe couplings and connectors) or applications utilizing only a fraction of the operation range available. In spite that these applications satisfy condition 5, the development of these products is based on major research activities and testing efforts for a specific application. Therefore, these products cannot be used as a foundation for development of new products. This implies that condition 5 by itself is not a guarantee for a mature technology.

In most applications utilizing the SMA technology, the sixth condition is not satisfied. The condition comprises a wide range of matters during the lifetime of the product. It regards environmental influences as well as operational conditions, degradation and retirement of the product. Regarding the operation and degradation, the complex behavior of the SMA is too difficult to control in a reliable way unless utilizing advanced feedback control (i.e. by using position and force sensing detectors). 
It follows that the SMA technology is still not sufficiently mature to be considered as a sensitive choice of technology when designing new products. This statement is supported by the findings from section 2 , stating that poor material property information might result in a rejection of technical solutions based on the shape memory effect already in the conceptual design phase. Also, the results from the international survey indicate that the use of SMAs in engineering design normally involves inefficient techniques based on trial and error and that the alloys to be utilized often are developed in parallel with the development of the product. On the other hand, the findings above provide fundamental guidelines on how the SMA technology should be developed so as to provide a safe and realistic alternative to other technologies.

\subsection{Recommended actions}

Provided that the requirements $I_{1}, I_{2}, P_{2}, M_{2}, D_{1}, D_{2}, S_{1}$ and $S_{2}$ in table 1 are fulfilled, the SMA technology will satisfy all the conditions constituent of a mature technology. These requirements are discussed in more detail below.

An essential activity, occurring throughout the engineering design process, concerns design analysis with respect to material properties, dimensions, form and expected performance. No analysis can be performed unless reliable material properties $\left(\mathrm{I}_{1}\right)$ and proper material behavior models, such as reliable constitutive equations $\left(\mathrm{P}_{2}\right)$, are available. Obviously, the material property information must include all aspects that might affect the functional as well as structural behavior. For instance, the influence of shape, dimension and thermomechanical history has to be fully documented.

Items $\mathrm{I}_{1}, \mathrm{P}_{2}, \mathrm{M}_{2}, \mathrm{~S}_{1}$, and $\mathrm{S}_{2}$ are highly mutually dependent. The material properties cannot be considered reliable and constitutive equations are not relevant unless the demands on stable material properties $\left(\mathrm{M}_{2}\right)$, standardized materials $\left(S_{1}\right)$ and standardized methods for testing phenomenological properties $\left(S_{2}\right)$ are satisfied. This indicates that the need for research that provide materials with stable and standardized material properties, as well as test methods for testing phenomenological properties, is more urgent than efforts to provide a wide range of commonly available materials $\left(\mathrm{M}_{1}\right)$. Standardization should be made gradually, starting with the test methods and a few materials suitable for the most frequent applications. If metallurgical aspects obstruct the possibility to develop more stable materials, more research must be directed towards controlling the behavior of the application during the life of operation $\left(\mathrm{O}_{1}\right)$.

Before the fifth condition set out by the technology readiness assessment can be satisfied, more detailed descriptions of product development projects must be made available in literature $\left(I_{2}\right)$. Obviously, it is better to disclose some competition advantages than to risk the entire technology to fade out due to bad experiences. This is also valid for item $\mathrm{D}_{2}$, termed design guidelines. Such guidelines should be based on experiences gained from projects involving utilization of SMAs.

An important condition for the designer to utilize design tools $\left(D_{1}\right)$ is that these are compatible with general design procedure models, design methods and design techniques. Such models, methods and techniques do not involve utilization of immature technologies. In the light of the discussion above, it might be argued that the need for design tools is less urgent than the need for standardized materials with relevant and reliable material properties. Further, although the requirements $I_{3}, P_{1}$ and $S_{3}$ are of less fundamental importance than the ones outlined above, these might serve as good guidelines for the future development of a more effective and efficient SMA technology.

Provided that the fundamental requirements outlined above are satisfied, the designer will be able to analyze concepts based on the shape memory effect and evaluate these against concepts based on other physical effects.

The establishment of a better knowledge in early design phases cannot be limited to the shape memory element alone - it must also include upgraded knowledge about the components with which the element is to interact. For instance, if a tube coupling device is to be designed for a corrosive environment, selection of a working principle based on the shape memory effect also stipulates the type of tube material, which must be compatible with the element with respect to corrosion resistance.

If a working principle based on the shape memory effect is chosen without first upgrading the knowledge about the interacting parts, information gained in the latter design phases might prove that the working principle proposed is not satisfactory and must be changed through a time-consuming iteration to the design phase in which the working principle originally was established. This shows that the problems of utilizing SMAs in engineering design do not only revolve around the SMA itself, but rather around a wide range of effects influencing both the SMA and its design environment. Consequently, when utilizing a SMA, the designer al- 
ways must start at a very detailed level of knowledge. This fact cannot be overcome by introducing new design procedure models, as indicated in $\mathrm{D}_{3}$. This requirement therefore should be considered as an expression of frustration rather than a foundation for future research.

\section{CONCLUSIONS}

Utilization of SMAs in engineering design has been studied from the viewpoint of engineering design and an international survey. In the light of a common evaluation technique of the maturity of new technologies, it is shown that the SMA technology is still not sufficiently mature to be utilized on a broad base for engineering design purposes. It is shown that to upgrade the SMA technology to a mature technology, major research efforts must be aimed at providing:

- materials with stable, reliable and standardized material properties, as well as constitutive equations, in a form that fulfills the needs of the engineering designer,

- literature offering more detailed descriptions of product development projects in which SMAs successfully have been utilized,

- design tools describing the functional behavior of SMAs,

- special design guidelines on how to handle the complex behavior of SMAs, and

- standardized methods for testing phenomenological properties.

The results of the study support the fact that research on SMAs so far mainly has been conducted with focus on metallurgical properties. Moreover, it is concluded that the conditions for utilizing SMAs must be improved through a closer collaboration between researchers from the fields of material technology and engineering design.

\section{Acknowledgments}

The authors wish to express their sincere thanks to Dr. Peder Andersson, Assoc. Prof. Robert Bjärnemo and Assoc. Prof. Åke Burman for valuable discussions and advice.

\section{References}

[1] Ölander A., J. of Am. Chem. Soc., 54 (1932), pp. 3819-3833.

[2] Abrahamsson P., Material med unika funktionella egenskaper, minnesmetaller, nya produkter (Department of Machine design, Lund Institute of Technology, Lund, 1993. In Swedish).

[3] Abrahamsson P. and Bjärnemo R., "The need for product design tools in shape memory technology", Third IUMRS International Conference on Advanced Materials, Sunshine City, Tokyo, August 30 to September 4, 1993, (Trans. Mat. Res. Soc. Jpn., 18B, 1994) pp.1171-1174.

[4] Bjärnemo R. and Burman A., "On the implementation of shape memory effects in a general FE-code", Third IUMRS International Conference on Advanced Materials, Sunshine City, Tokyo, August 30 to September 4, 1993, (Trans. Mat. Res. Soc. Jpn., 18B, 1994) pp.1175-1178.

[5] Møster E., On the concept for a down-hole-ball releasing unit (Department of Machine design, Lund Institute of Technology, Lund, 1997).

[6] Burman $\AA$., Møster E. and Abrahamsson P., "On the influence of functional materials on engineering design", To be published.

[7] Asimov M., Introduction to design (Prentice Hall, Englewood Cliffs, New York, 1982).

[8] Hubka V. and Eder W.E., Engineering design (Heurista, Zürich, 1992).

[9] Olsson K.G.F., Systematisk konstruktion (Department of Machine Design, Lund Institute of Technology, Sweden, 1976. PhD thesis. In Swedish).

[10] Pugh S., Total design (Adison-Wesley Publishing Company, Great Britain, 1991).

[11] Pahl G. and Beitz W., Engineering design (Springer-Verlag, The Design Council, Berlin, London, 1995).

[12] Ullman D.G., The mechanical design process (McGraw-Hill, Singapore, 1992). 